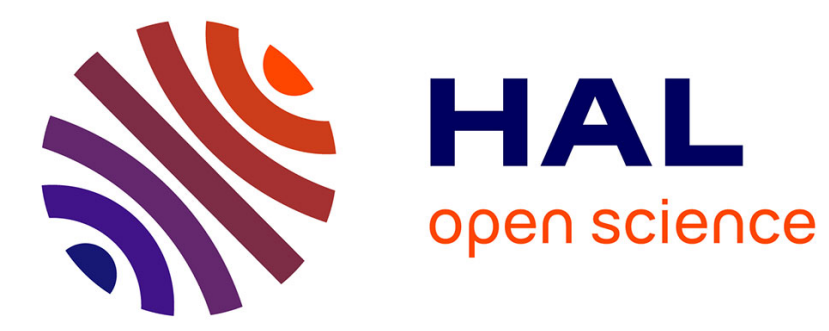

\title{
Endpoints in paediatric oncology
}

Paolo Paolucci, Vera Cioni, Elena Bigi, Laura Lucaccioni, Carmen Cano

\section{To cite this version:}

Paolo Paolucci, Vera Cioni, Elena Bigi, Laura Lucaccioni, Carmen Cano. Endpoints in paediatric oncology. European Journal of Clinical Pharmacology, 2010, 67 (S1), pp.33-40. 10.1007/s00228-0100923-1 . hal-00642438

\section{HAL Id: hal-00642438 \\ https://hal.science/hal-00642438}

Submitted on 18 Nov 2011

HAL is a multi-disciplinary open access archive for the deposit and dissemination of scientific research documents, whether they are published or not. The documents may come from teaching and research institutions in France or abroad, or from public or private research centers.
L'archive ouverte pluridisciplinaire HAL, est destinée au dépôt et à la diffusion de documents scientifiques de niveau recherche, publiés ou non, émanant des établissements d'enseignement et de recherche français ou étrangers, des laboratoires publics ou privés. 


\section{Endpoints in Paediatric Oncology}

Authors: Paolucci P, Cioni V, Bigi E, Lucaccioni L, Cano MC

Department of Mother and Child

University of Modena and Reggio Emilia

Via del Pozzo, 71

41100 Modena, Italy

Corresponding author: Prof. Paolo Paolucci, MD

Department of Mother and Child

University of Modena and Reggio Emilia

Via del Pozzo, 71

41100 Modena, Italy

Tel.: +39059422 2535

Fax: +39 0594224268

E-mail: paolo.paolucci@unimore.it

Part of TEDDY Supplement 


\section{Abstract}

\section{Purpose}

The purpose of this review is to address the issue of endpoints in paediatric oncology. Oncologists use this term to refer to an outcome they are trying to measure with a clinical trial, which may become accordingly the object of scientific articles. The outcome measured may concern both efficacy and safety, although in different perspectives.

\section{Methods}

Based on both literature and experience developed in clinical trials, the different types of endpoints have been critically analysed in their power to provide the highest information of therapeutic interest (efficacy and safety) with the least risk and discomfort for the individual. Endpoints have been distinguished in primary, secondary and surrogate ones. The most relevant differences have been discussed in comparison with adult oncology settings of endpoints.

\section{Results}

The rarity of cancer in childhood and adolescence and the objective difficulty of enrolling statistically conceivable numbers of individuals have determined the utmost positive development of large scale, multinational clinical trials. The most interesting consequence is that the impact of multiplicity interferences, which is usually present in virtually all clinical trials developed for adults with cancer, is not a common event in paediatric oncology. Nevertheless, many of the questions concerning the different impact on outcome and survival of clinical trials developed in adult oncology remain unanswered due to the objective limitations still existing in terms of cure as compared to paediatric oncology. The powerful consistency of cure rate, as the most relevant end point of clinical trials developed in paediatric oncology, addresses additional considerations to support the relevant differences existing between adult and paediatric oncology: both the development of clinical trials with different aims (confirmatory versus primary response) and the limited impact of multiplicity limitations may determine different implications about the meaning of endpoints in paediatric and adult oncology.

\section{Conclusion}

The aim of cancer treatment is to improve survival (SUR) and quality of life (QoL), but some restraints on the conduct of clinical trials may make these goals unattainable. Clinical trial endpoints represent a measure method aimed to grant answers to questions addressed by the clinical trial itself. The effect of the new regulation is expected to stimulate highquality research and provide robust information on paediatric drugs to increase the availability of such drugs to children.

\section{Keywords}

Endpoint - clinical trials - paediatric oncology 


\section{Endpoints in Paediatric Oncology}

\section{Introduction}

The aim of cancer treatment is to improve survival (SUR) and quality of life (QoL), but some restraints on the conduct of clinical trials may make these goals unattainable. The majority of childhood malignancies consists of rare conditions that uniquely affect this population. A large percentage of paediatric patients in Europe affected by these conditions are treated within the context of not-for-profit clinical trials conducted by co-operative groups. Nevertheless, promising new agents are not made available at an early stage in their development and their use cannot be studied for such conditions without unnecessary delays in the start of evaluation in the paediatric population [1]. In such cases, clinical development should begin early in the paediatric population, following assessment of initial safety data and reasonable evidence of potential benefit. Also negative effects of anticancer drugs on the developing human body can cause serious "late effects" in survivors of childhood cancer. This further stresses the need to develop more specific anti-cancer drugs with less (short and long term) side effects and the need for long term follow up (FUP) of cancer survivors and pharmacovigilance [2,3].

In paediatric settings, clinical trials may be difficult to recruit large numbers of patients as many diseases affect only few thousands or even fewer than one hundred children in Europe (EU). Accordingly, conduct, analysis, and interpretation of studies in rare conditions at times may be constrained to varying degrees by the prevalence of the disease. Of course, no methods exist that are relevant to small studies that are not also applicable to large studies [4]. In addition, few formal studies have taken place on the pharmacology of many drugs in children, and even fewer trials sponsored by drug companies have aimed to show the anti-tumour efficacy of these drugs against cancers specific to childhood in order to support a licensed indication: less than $15 \%$ of drugs approved for adults with cancer and less than $50 \%$ of those commonly used have a role in paediatric oncology. In order to overcome this limitations, paediatric oncologists have been developing over the last 30 years large scale, collaborative, mainly non-commercial, prospective clinical trials and improvements in supportive care, which were highly successful in term of results as by the year 2000 about $75 \%$ of newly diagnosed children with cancer were expected to be cured.

Experimental data for cancer treatment are derived mainly from efficacy trials with the main aim of improving survival for a particular type of cancer. Even when these trials are of good quality, they are not always done with a view to submit the data to the Regulatory Authorities, leading to many drugs being used off-label, especially in the paediatric population. The need to undertake appropriate clinical trials for registration studies in children was taken into consideration in the context of the European Directive EC/2001/20 on Good Clinical Practice [5], in which prominence is given to the ethics involving inclusion of children in experimental populations.

Although data from the not-for-profit trials might be collected in a form suitable for use by regulatory authorities, without the support of an industrial partner interested in registering a paediatric indication, the data will not be used to improve labelling of drugs for paediatric use. The new Regulation [6,7] provides a non-mandatory tool, namely a new type of marketing authorisation for off-patent drugs called Paediatric Use Marketing Authorisation (PUMA), which covers therapeutic indications relevant for use in the paediatric population, as well as appropriate formulations. On the contrary, the Paediatric Investigational Plan (PIP) represents the mandatory tool for new marketing authorization (patent) and new indications, administration routes and formulations relevant for use in the paediatric population concerning already patented substances.

\section{Study endpoints}

Clinical trial endpoints represent a measure method aimed to grant answers to questions addressed by the clinical trial itself. They may be different and serve different purposes, such as the measure of disease activity or clinical benefits in various clinical settings. Oncologists use the term endpoint to refer to an outcome they are trying to measure with a clinical trial, which may become accordingly the object of scientific articles [8]. Endpoints can include all kinds of things, those related to the effectiveness of treatment as well as some other ones. However, the endpoints selection must take into account the need of obtaining the highest information of therapeutic interest with the least risk and discomfort for the individual. Ethical Committees must control that endpoints are pertinent to the objective of the study and represent the most effective way for the assessment of the pharmacological response. In terms of safety, non invasive methods (e.g. mortality, QoL, incidence of events, response rate, cure rate, relapse rate, imaging techniques, etc.) should be preferred to invasive procedures (biopsies, catheters, endoscopies, etc.), unless the latter ones are mandatory for the evaluation of treatment efficacy or when they represent the routine monitoring of the disease course according to the rules of good clinical practice.

Of course, this scenario is even more complex when we deal with children's pathologies for a number of specific reasons we shall attempt to focus on herewith. For instance, at variance of conventional oncology drug development, early phase clinical trials concerning children cannot evaluate safety only as in adults, but should provide also some evidence of biological drug activity, such as tumour shrinkage. Endpoints for later phase efficacy studies commonly evaluate whether a drug provides a clinical benefit such as prolongation of survival or an improvement in symptoms. Of course, concerning children and adolescents, later phase studies should take into account QoL and its preservation for lifetime besides the 
control of disease and symptoms, but, as we shall discuss herewith, this represents a rather complex and not easily solvable problem when we refer to long term toxicity. Differences between adults and children do have implications also in the contents of the direct relationship to establish with the patients. However, no differences exist amongst the response categories (complete, partial, objective, minor, stable disease, progressive disease, etc.) between adults and children with cancer.

Patients' response is characterized by the greatest amount of shrinkage they achieve from the time treatment started. So if a patient has a major shrinkage which is a response but later has tumour growth it is still categorized as a response. The duration of the response is from the time response is achieved until renewed growth is detected. The response criteria should be predefined in the protocol before the start of the study. Endpoints are distinguished in primary, secondary and surrogate ones. The former should make focus on the principal objective of the study and determine the size of the sample to be analysed from a statistical point of view.

\section{Primary endpoints}

Increased survival and symptomatic improvement represent the essential clinical benefits we may identify as primary or clinical endpoints in paediatric and adult oncology. Clinical benefits support drug approval as they represent the most important result to be achieved by the use of any substance although they include also effects on other established secondary and surrogate endpoints[9]. However, concerning paediatric oncology the contexts where to use clinical, secondary and/or surrogate endpoints may be different when compared to adults, as the vast majority of anti-cancer drugs employed to cure children and adolescents have never been part of any official drug approval procedure specifically aimed for the paediatric ages.

Under these circumstances, paediatric oncologists developed clinical trials under an unendorsed, unofficial regimen projected to treat serious and life-threatening diseases for which they were able to demonstrate an improvement over available therapy or provide therapy where none existed. As mentioned before, this historical limitation is going to be overcome thank to the new regulation in Europe. Nevertheless, originally the situation was that clinical trials in paediatric oncology were inevitably based more on an effect that was reasonably likely to predict clinical benefit (based on epidemiologic, therapeutic, pathophysiologic, or other expected evidences) than on a formally established primary endpoint; this situation might resemble and be compatible with that of a surrogate endpoint. Indeed, the majority of anti-cancer drugs have been used off-label in paediatric oncology so far and several of these substances have only recently attained in some Europe Member States the label status based on the number and the quality of data developed by paediatric oncology trials over the last three decades. However, many other substances are still used off-label and no manufacturer has offered so far to conduct specific clinical trials aimed to obtain a PUMA, according to the above mentioned new regulation. In fact, due to a high rate of responses in paediatric oncology, evidence from a limited number of trials may be sufficient to support an efficacy supplement for treatment of a different stage of the same cancer or even of a different tumour. All these situations seem to resemble what goes under the definition of the term regular approval, which denotes the longstanding route of drug approval based on the demonstration of clinical benefit as opposed to the term accelerated approval, which is associated with use of a surrogate endpoint that is reasonably likely to predict benefit.

Primary endpoints may be based on both tumour measurements or tumour assessments; tumour endpoint assessments generally should be verified by central reviewers blinded to study treatments. This measure is especially important when the study itself is not blinded and it may be appropriate for the scientific committee ruling over the trial to audit a sample of the scans to verify the central review process. Centralized independent proof of tumour endpoint assessments (especially for progression free survival or disease free survival) may not be necessary when randomized trials are blinded.

Once we understand that clinical trials developed for children with cancer were not meant to support cancer drug approval in the vast majority of the cases, no major differences exist between adults and children primary endpoints in principle. However, differences may still exist concerning the achievability of results investigated by a primary endpoint in a given population (e.g. due to the poor prognosis of the disease) and the meaning of the primary endpoint due to the objective differences in terms of curability of cancer between adults and children.

Examples of primary endpoints focusing on response, control of disease, survival are the following:

Objective Response (OR) means measuring tumour shrinkage, tumour size reduction of a predefined amount and for a minimum time period, as determined by tumour assessments from radiological tests or physical examinations. Response duration usually is measured from the time of initial response until documented tumour progression. When OR is defined as the sum of partial responses plus complete responses, it is a direct measure of drug anti-tumour activity, which can be evaluated in a single-arm study. Stable disease should not be a component of OR as it can reflect the natural history of disease, whereas tumour reduction is a direct therapeutic effect. Also, stable disease can be more accurately assessed by time to progression or progression free survival analysis (see below). As cancers hardly ever get smaller without treatment, a significant tumour shrinkage shows treatment is having an effect on the tumour. Response is usually the primary endpoint in Phase II trials, but is often measured in Phase I and III trials as well, especially in paediatric oncology. A high, substantiated OR can support regular approval in select solid tumours, but also response duration, relief of tumour-related 
symptoms, and drug toxicity should be considered when making the approval decision (composite endpoints). Of course, response is not an endpoint for adjuvant clinical trials where the primary tumour has been removed surgically since in that case there are no detectable tumours to measure.

Overall Survival (OS) is defined as time from randomization, or front-line therapy start, until death from any cause, which is measured in the intent-to-treat population. Apparent differences in outcome between historical controls and current treatment groups can arise from differences other than drug treatment (patient selection, improved imaging techniques, improved supportive care). Randomized studies minimize the effect of these differences by providing a direct outcome comparison. Demonstration of a statistically significant improvement in overall survival can be considered to be clinically significant if the toxicity profile is acceptable; it may often support new drug approval. Difficulties in performing and analyzing survival studies include long term FUP periods in large trials and subsequent cancer therapy potentially confounding survival analysis in adult population. On the opposite, in paediatric oncology these issues may be overcome due to both a much better chance of response and the feasibility of long term FUP in children and adolescents for obvious reasons.

Median Overall Survival (MOS), or Median Survival Time (MST), is the time from either diagnosis or treatment at which half of the patients with a given disease are found to be, or expected to be, still alive. In a clinical trial, MOS may be one possible way to measure how effective a treatment is whenever a randomized trial is not feasible. MOS may be useful in conditions such as high risk brain tumours with very limited response chance to treatment (SUR $<6$ months) in view of measuring a potential SUR gain in comparison with historical controls. In all these situations, functional scales for testing ability (QoL) may be helpful to power the comparison between series.

Disease-Free Survival (DFS) is defined as time from randomization, or front-line therapy start, until recurrence of tumour or death from any cause. The most frequent use of this endpoint is in the adjuvant setting after definitive surgery or radiotherapy. DFS also can be an important endpoint when a large percentage of patients achieve complete responses with chemotherapy, such as in paediatric oncology. However, bias can be introduced if the frequency of long term FUP visits is dissimilar between the study arms or if dropouts are not random because of toxicity. Some analyses count cancer-related deaths as DFS events and censor non-cancer deaths. This method can introduce bias in the attribution of the cause of death. Furthermore, any method that censors patients, whether at death or at the last visit, assumes that the censored patients have the same risk of recurrence as non-censored patients. For these reasons, paediatric oncologists prefer to refer to:

Event-Free Survival (EFS) is defined as time from randomization, or front-line therapy start, until objective tumour progression, secondary malignancy or cancer related death.

Time to Progression (TTP) and Progression-Free Survival (PFS) have served as primary endpoints for drug approval [J]. TTP is defined as the time from randomization until objective tumour progression; it does not include deaths. PFS is defined as the time from randomization until objective tumour progression or death and thus can be a better correlate to overall survival. The precise definition of tumour progression is important and should be carefully detailed in the protocol. Cancer trials are often small, and proven survival benefits of existing drugs are generally modest. The role of PFS as a primary endpoint to support licensing approval varies in different cancer settings. Whether an improvement in PFS represents a direct clinical benefit or a surrogate for clinical benefit depends on the magnitude of the effect and the risk-benefit of the new treatment compared to available therapies. It is a very appropriate primary endpoint in paediatric solid tumours.

Time-to-Treatment Failure (TTF) is defined as a composite endpoint measuring time from randomization to discontinuation of treatment for any reason, including disease progression, treatment toxicity, and death. TTF does not adequately distinguish efficacy from these additional variables. A regulatory endpoint should clearly distinguish the efficacy of the drug from toxicity, patient or physician withdrawal, or patient intolerance. Of course, it is not used in paediatric oncology, due to its inadequate distinction amongst efficacy and other additional variables.

Additional primary endpoints may be focused on the effects of randomizations between two or more different drugs, different pharmaceutical preparations of the same drug, two or more different doses of the same drug, two or more different timing of administration of the same drug, two or more different modalities of administration (continuous vs pulses) of the same drug, two or more different ways of administration of the same drug.

In conclusion, a primary endpoint is defined as a clinical endpoint that provides evidence sufficient to fully characterize clinically the effect of a treatment in a manner that would support a regulatory claim for the treatment. Because evaluation of the impact of treatment on a primary endpoint is the major purpose of a clinical trial, the sample size of the trial is based upon the power of the trial to detect a specified clinical benefit on the primary endpoint.

\section{Secondary endpoints}

A secondary endpoint is a clinical endpoint that provides additional clinical characterization of treatment effect, but it is not sufficient to characterize fully the benefit or to support a claim for a treatment effect [11]. By definition, a secondary endpoint could not, by itself, be convincing of clinically significant treatment effects, even if it were observed to be statistically significant. Defined in this way, a secondary endpoint could not become a primary endpoint as secondary endpoints cannot be validly analyzed if the primary endpoint does not demonstrate clear statistical significance. This 
distinction in definitions does not, however, illustrate why controversy exists concerning whether a statistically significant secondary endpoint should be considered valid. Likely, the controversy arises when there is a multiplicity of endpoints whose collective use has not been considered in advance and when none of these endpoints may fully characterize a treatment effect. In clinical trials protocols, a number of secondary variables for efficacy may be present, but up to now, no common consent exists about the nature, the role and the weight of secondary endpoints in clinical trials especially in paediatric oncology. On the opposite, in adult clinical trials, secondary endpoints may provide additional clinical characterization of treatment effects and may be related to both tumour factors and host factors which may have positive implications in terms of QoL[12] during the treatment course. Otherwise, the most significant effects can be considered for additional claim once the primary objective of the clinical trial has been achieved and if they are part of the confirmatory strategy.

However, changes in secondary variables that are considered a direct consequence of the respective changes in the primary variables cannot be part of the labelling claims. For reasons mentioned before, we believe that the new regulation as soon as it will enter fully into its operative phase, clinical trials in paediatric oncology will be dealing with both primary variables, as the most important ones (mortality, cure percentages, survival, etc.), and secondary endpoints focusing on additional variables which also deserve attention as it is for clinical trials developed for adult pathologies. In addition, it is conceivable that variables, having the potential of being indicative of major clinical benefit or present an important safety issue (e.g. mortality) will not be relegated to a secondary variable condition due to the too small (power too low) size of the planned trial as it may happen in paediatric clinical oncology. Under the past circumstances, the major attitude of clinical trials in paediatric oncology has been that to focus on the strongest and most important primary variables mentioned above, unless we were dealing with an uncontrolled single institution, small size pilot, exploratory study.

Secondary endpoints may include many different variables, which may be (directly or not) related to:

- disease such as relapse (site, type, time, etc.) or mortality during treatment;

- treatment such as costs due to duration of hospitalization, supportive therapies impact (hemoderivates, nutrition, emesis, pain, etc.), infections (anti-microbial agents, etc.), clinical management (laboratory tests, imaging, etc.), etc.;

- education such as days of absence from school or school performances;

- $\quad$ social costs such as family management (accommodation, transportation, etc.);

It may appear questionable if primary or secondary endpoints should include symptom assessment and/or physical signs representing symptomatic improvement (weight gain, decreased effusion, pain, etc.). Indeed, they should be considered strictly clinical benefits as measures of global QoL have not served for oncology drug approvals and instruments measuring QoL may simply indicate less toxicity rather than effectiveness. In addition, clinical benefits are not able to distinguish between improvement in tumour symptoms and lack of drug toxicity [13].

Clinical benefits are prevalently considered as endpoints in adult oncology, because they may not always be predicted by, or correlate with, OR. They should be based on more direct evidence of improvement in survival, above and beyond improvement in a patient's QoL (due to control of both tumour and host factors), improved physical functioning (due to control of tumour factors), or improved tumour-related symptoms (due to control of tumour factors). Clinical benefit is an informal term which usually means anything other than progressive disease, but the use of this term is "suspect", because it is not automatically clear that patients with stable disease are benefiting from treatment since the natural history of cancer can include periods of apparent stable disease and since tumour shrinkage is not equal to clinical benefit to begin with. When we see this term, which is not used currently in paediatric oncology, we should look at both the CR and PR rates and also the duration of "benefit" including for stable disease cases. In fact, durable complete response is considered an established endpoint of clinical benefit in acute leukemia in children, where complete response is associated with less infection, bleeding, and blood product support (composite endpoints).

\section{Confirmatory trials}

In adult oncology confirmatory trials are mainly referred to secondary endpoints and should demonstrate that the investigational product provides at least clinical benefit which confirms results of previous trials [14]. There should thus be sufficient evidence available demonstrating that the chosen primary endpoint can provide a valid and reliable measure of clinical benefit in the patient population described by the inclusion criteria. On the opposite, in paediatric oncology, for reasons mentioned above, clinical trials are less frequently of confirmatory meaning. Instead, they are optimisation/ superiority or non-inferiority clinical trials as their main endpoints are OS, EFS or PFS (depending on the type of tumour) e.g. endpoints investigating both cure of cancer and ultimately QoL. Briefly, superiority trials are aimed to provide direct evidence of treatment improvement as opposed to the indirect evidence which may be provided by non-inferiority trials, although the latter trials may be taken into account anytime a major break through is not expected and no step backwards may be legitimate. 


\section{Surrogate Endpoints}

According to Prentice (1989) [15], surrogate endpoint literally means to substitute for. Therefore, in the simplest terms a surrogate endpoint is a measurement that can be substituted for a true endpoint to predict either benefit (e.g., survival) or harm (e.g., late toxicity). However, any changes induced in the surrogate endpoint by a treatment must accurately reflect changes in the true endpoint. Clearly, this is an area which requires further clarification. In paediatric oncology a number of biological and bio-molecular markers are potentially eligible to be considered as valid surrogate endpoints :

- immunophenotyping;

- tumour markers (CAE, CA19.9, $\alpha$ fetoprotein, $\beta$ human chorionic gonadotropin, neuron specific enolase, vanilmandelic acid, etc.);

- cytogenetics;

- $\quad$ specific mutations in cancer-related genes;

- $\quad$ time and level of minimal residual disease;

- gene profile expression;

- polymorphisms;

- pharmacogenomics;

- cellular and nuclear phenomena (proliferation, apoptosis, DNA ploidy);

- tumour characteristics detected by functional imaging (MR, PET).

Usually, biomarkers assayed from blood or body fluids have not served as primary endpoints for cancer drug approval. Research is in progress to establish the validity of available tests and determine whether improvements in biomarkers predict clinical benefit for all cancers of paediatric age [16]. In addition, it is still questionable if the treatment is able to modify the course of the biology of cancers. Therefore, biomarkers are useful as prognostic factors and helpful in patients selection as well as stratification factors to be considered in study designs. All these issues do represent the milestones of the development of clinical trials in paediatric oncology as they fully fulfil the criteria needed to be considered as an appropriate surrogate endpoint: the association with the true endpoint biologically; the association of treatment with the potential surrogate endpoint; the potential surrogate mediation of the effect of the treatment on the true endpoint. It is conceivable that the likelihood of finding useful surrogate endpoints in paediatric oncology is greatly enhanced by a situation in which the disease pathogenesis and mechanisms of action of the treatment are well experienced and understood. In paediatric oncology (both leukemias and some solid tumours), potential surrogate endpoints are integrated into studies with well-defined true clinical events as primary endpoint. Therefore, there is a consistent chance to accomplish that a perfect surrogate would be one where all of the effect of treatment on the true endpoint is mediated through the surrogate endpoint.

There are several potential advantages of using surrogate endpoints in clinical trials, especially in phase III trials, but they may often be time consuming and expensive. They may also be attractive in phase II trials to help provide an estimate of the effect of the new treatment under investigation and allow introduction of effective treatments at an earlier stage or rejection of treatments with no benefit before embarking on phase III trials. In addition, the use of surrogate endpoints in early clinical trials may assist to better select patient or tumour categories that are most likely to benefit from new treatments allowing a reduction of the sample size in randomised trials.

Surrogates may also be used in a situation where we have competing risks or the true endpoint is complicated by the use of other treatments, a common situation in trials of cancer treatment. In certain studies, surrogate endpoints or biomarkers might be useful in establishing the mechanism of action of a treatment or provide better understanding of the underlying mechanism of the disease process. Finally, surrogate endpoints may be used in a broader context, not only for assessment of the benefits of a treatment but also as indicators of late toxicity.

As mentioned above, a surrogate endpoint should totally capture any effect of the treatment. However, it is unlikely that any endpoint would capture both the beneficial and harmful effects of treatment. Disease-related surrogates will generally underestimate toxic effects and it may well be that a surrogate does not detect potential side-effects of a treatment which would render a new treatment unacceptable. However, as different treatments are likely to work through different mechanisms and the effect of a treatment is likely to be different in different malignancies, the applicability of a surrogate endpoint from one situation to another must be considered carefully. It is likely that a panel of surrogate endpoints will be required to capture fully the complex nature of any disease-treatment interaction.

\section{Questions and differences between children and adults with cancer}

Many of the questions concerning the different impact on outcome and survival of clinical trials developed in adult oncology remain unanswered due to the objective limitations still existing in terms of cure as compared to paediatric oncology. These include: what is the optimal recipe for the treatment of advanced disease, are trial results applicable to the 
broad population of specific cancer patients, is there a worthwhile impact on survival, what is the role of second-line therapy and how can we reliably assess new agents for use in cancer therapy innovation. If we take into account that most of these questions have a largely positive answer, with the only exception of the last one concerning the role of new agents for reasons mentioned above, we can understand the reasons why the meaning of endpoints in paediatric oncology differ from adult. These differences are strongly explained by the observation that the endpoints of clinical trials developed in paediatric oncology are prevalently aimed to fulfil two results: more cure and less toxicity (mainly long term one). This is the reason why in paediatric oncology clinical benefits may receive less attention than in adult trials and even less when these benefits are not co-expression of tumour shrinkage, disease control and measurable improvement of EFS, OS, PFS. Therefore, confirmatory trials, based mainly on secondary endpoints and aimed to confirm results obtained in previous trials are less frequent in paediatric oncology, because trials are mainly either aimed to ameliorate EFS (optimization/superiority trials to improve the cure rate) or to reduce long term toxicity due to the treatment (non-inferiority trials to reduce toxicity without reduction of EFS). The key difference is that in paediatric oncology the obvious expectation that responders do survive longer than non-responders and the decrease in tumour size is suggestive of biological activity give evidence that response to treatment has prognostic value for survival over and above that of prognostic factors known at the time of starting therapy. The strong expectation of personalized treatment of children with cancer goes along with this concept.

In oncology clinical trials, endpoints may be the same for adults and children. However, in paediatric oncology their meaning may be different as a consequence of the little impact, if any, they had on registration procedures so far. In addition, in paediatric oncology we have few, if any, confirmatory trials for registration as apposed to "response" trials, in which disease progression and recurrence are typically assessed based on objective findings such as various imaging techniques together with objective chemical, biochemical and biomolecular criteria which are conventionally used to assess progression. As the aim of the clinical trials is the cure of the children with cancer, lot of attention has been dedicated to the FUP, but few formal studies have taken place on the pharmacology of these drugs in children, and even fewer trials sponsored by drug companies have aimed to show the anti-tumour efficacy of these drugs against cancers specific to childhood in order to support a licensed indication.

This historical attitude has generated both positive and negative consequences. The former ones are: the study outcome of patients is largely available (off-therapy registries) and adequate to assess the expected treatment effect in a balanced way; withdrawal from treatment prior to scheduled completion is very rare as well as lost to FUP; change treatment before evidence of progression or present with missing evaluations followed by evidence of progression is exceptional. The negative consequences currently existing both in Europe and the USA have been mentioned in the introduction. When using such drugs in children, potential acute and chronic effects should take into account changes during the developmental stages from infancy to adulthood. Physiological and psychological changes might also occur due to the effect of treatments that inhibit the growth of tissues and organs (e.g. radiation and certain drugs).

The effect of the new regulation is expected to stimulate high-quality research and provide robust information on paediatric drugs to increase the availability of such drugs to children. This regulation aims: to keep ineffective treatment, incorrect dosing, and adverse drug reactions to a minimum; to reduce hospitalisations and deaths; to improve QoL; to provide economic benefits. European regulatory actions are important steps to develop specific paediatric dosing recommendations at an early stage in the development process and to improve the safety of new anticancer drugs in children.

Due to the rarity of cancer in childhood and adolescence and the objective difficulty of enrolling statistically conceivable numbers of individuals, which has determined the utmost positive development of large scale, multinational clinical trials, the impact of multiplicity interferences, present in virtually all clinical trials, is not common in paediatric oncology. Regardless this limitation, the powerful consistency of cure rate, as the most relevant end point of clinical trials developed in paediatric oncology, addresses additional considerations to support the differences between adult and paediatric oncology in terms of results. Multiplicity can have a substantial influence on the rate of false positive conclusions which may effect approval and labelling of an investigational drug, which has not been a frequent goal of clinical trials in paediatric oncology so far and, therefore, with little or no influence on results of the trials in the paediatric field. Therefore, both the development of clinical trials with different aims (confirmatory versus primary response) and the limited impact of multiplicity limitations may determine different implications about the meaning of endpoints in paediatric and adult oncology. 


\section{Acknowledgment}

This contribution is part of the Task-force in Europe for Drug Development for the Young (TEDDY) Network of Excellence supported by the European Commission's Sixth Framework Program (Contract n. 0005216 LSHBCT-2005-005126). 


\section{Conflict of interest}

The authors declare that they have no conflict of interest. 


\section{References}

1) EMEA. Committee for medicinal products for human use. Note for guidance on evaluation of anticancer medicinal products in man - Addendum on paediatric oncology; London, 24 July 2003. http://www.emea.europa.eu.int CPMP/EWP/569/02. Accessed 15 March 2010

2) Oeffinger KC, Mertens AC, Sklar CA, Kawashima $T$, Hudson MM, Meadows AT, Friedman DL, Marina N, Hobbie W, Kadan-Lottick NS, Schwartz CL, Leisenring W, Robison LL for the Childhood Cancer Survivor Study (2006) Chronic health conditions in adult survivors of childhood cancer. N Engl J Med 12; 355 (15):1572-82

3) Oeffinger KC, Hudson M M (2004) Long-term Complications Following Childhood and Adolescent Cancer: Foundations for Providing Risk-based Health Care for Survivors. CA Cancer J Clin 54;208-236

4) EMEA. Committee for medicinal products for human use. Guideline on clinical trials in small populations; London, 27 July 2006. http://www.emea.europa.eu.int Ref. CHMP/EWP/83561/2005. Accessed 15 March 2010

5) European Parliament and Council Directive 2001/20/EC on the approximation of the laws, regulations and administrative provisions of the Member States relating to the implementation of Good Clinical Practice in conduct of clinical trials on medical products for human use. Official Journal of the European Union 1.5.2000; L 121/34.

6) Regulation (EC) n. 1901/2006 of the European Parliament and of the Council of 12 December 2006 on medicinal products for paediatric use and amending Regulation (EEC) No 1768/92, Directive 2001/20/EC, Directive 2001/83/EC and Regulation (EC) No 726/2004. Official Journal of the European Union 27.12.2006, L378/1L378/19.

7) Regulation (EC) n. 1902/2006 of the European Parliament and of the Council of 20 December 2006 amending Regulation 1901/2006 on medicinal products for paediatric use. Official Journal of the European Union 27.12.2006, L378/20-L378/21.

8) CancerGuide. Endpoints: How the Results of Clinical Trials are Measured; http://www.CancerGuide.org/endpoints.html. Accessed 15 March 2010

9) Guidance for Industry. Clinical Trial Endpoints for the Approval of Cancer Drugs and Biologics. U.S. Department of Health and Human Services Food and Drug Administration. May 2007

10) EMEA. Committee for medicinal products for human use. Appendix 1 to the guideline on the evaluation of anticancer medicinal products in man (CHMP/EWP/205/95 REV.3). Metodological consideration for using progression-free survival (PFS) as primary endpoint in confirmatory trials for registration. London, 27 July 2006. http://www.emea.europa.eu.int CHMP/EWP/267575/2006. Accessed 15 March 2010

11) O’Neill RT (1997) Secondary Endpoint Cannot Be Validly Analyzed if the Primary Endpoint Does Not Demonstrate Clear Statistical Significance. Controlled Clinical Trials 18:550-556.

12) Roila F, Cortesi E (2001) Quality of life as primary endpoint in oncology. Ann Oncol 12: 3-6.

13) Sullivan R (2004) Clinical trial design in oncology. Lancet Oncol 5(12):759-763

14) EMEA. Committee for medicinal products for human use. Guideline on evaluation of anticancer medicinal products in man; London, 14 December 2005; http://www.emea.europa.eu.int CPMP/EWP/205/95. Accessed 15 March 2010

15) Prentice RL (1989) Surrogate endpoints in clinical trials: Definition and operational criteria. Statistics in Medicine $8,431-440$ 
16) Cooper R, Kaanders JHAM (2005) Biological surrogate end-points in cancer trials: Potential uses, benefits and pitfalls. Eur J Cancer 41 1261-1266 